\title{
Approximating Minimum Bounded Degree Spanning Trees to within One of Optimal
}

\author{
MOHIT SINGH, Microsoft Research, Redmond \\ LAP CHI LAU, The Chinese University of Hong Kong
}

In the Minimum Bounded Degree Spanning Tree problem, we are given an undirected graph $G=(V, E)$ with a degree upper bound $B_{v}$ on each vertex $v \in V$, and the task is to find a spanning tree of minimum cost that satisfies all the degree bounds. Let OPT be the cost of an optimal solution to this problem. In this article we present a polynomial-time algorithm which returns a spanning tree $T$ of cost at most oPT and $d_{T}(v) \leq B_{v}+1$ for all $v$, where $d_{T}(v)$ denotes the degree of $v$ in $T$. This generalizes a result of Fürer and Raghavachari [1994] to weighted graphs, and settles a conjecture of Goemans [2006] affirmatively. The algorithm generalizes when each vertex $v$ has a degree lower bound $A_{v}$ and a degree upper bound $B_{v}$, and returns a spanning tree with cost at most OPT and $A_{v}-1 \leq d_{T}(v) \leq B_{v}+1$ for all $v \in V$. This is essentially the best possible. The main technique used is an extension of the iterative rounding method introduced by Jain [2001] for the design of approximation algorithms.

Categories and Subject Descriptors: F.2.2 [Analysis of Algorithms and Problem Complexity]: Nonnumerical Algorithms and Problems-Computations on discrete structures; G.2.2 [Discrete Mathematics]: Graph Theory-Network problems, Trees

General Terms: Algorithms, Theory

Additional Key Words and Phrases: Approximation algorithms, spanning trees, bounded degree, iterative rounding

ACM Reference Format:

Mohit Singh and Lap Chi Lau. 2015. Approximating minimum bounded degree spanning trees to within one of optimal. J. ACM 62, 1, Article 1 (February 2015), 19 pages.

DOI: http://dx.doi.org/10.1145/2629366

\section{INTRODUCTION}

The Minimum Bounded Degree Spanning Tree (MBDST) problem is defined as follows: Given an undirected graph $G=(V, E)$, a cost function $c: E \rightarrow \mathbb{R}$ on the edges, and a degree upper bound $B_{v}$ on each vertex $v \in V$, find a spanning tree of minimum cost that satisfies all the degree bounds. When all degree bounds are two (i.e., $B_{v}=2$ for all $v$ ), the MBDST problem specializes to the Minimum Cost Hamiltonian Path problem, and thus even the problem of checking whether there exists a feasible solution is NP-complete. In unweighted graphs, Fürer and Raghavachari [1994] gave an elegant algorithm that returns a spanning tree in which the degree of each vertex is at most $B_{v}+1$, or returns a witness certifying that the degree bounds are infeasible. It was conjectured in Goemans [2006] that this result can be generalized to weighted graphs.

A preliminary version of this article appeared in Proceedings of the 39th ACM Symposium on Theory of Computing (STOC 2007).

Authors' addresses: M. Singh, Microsoft Research, 1 Microsoft Way, Redmond, WA 98004; L. C. Lau, Computer Science and Engineering, The Chinese University of Hong Kong, Shatin, Hong Kong. Correspondence email:mohits@microsoft.com.

Permission to make digital or hard copies of all or part of this work for personal or classroom use is granted without fee provided that copies are not made or distributed for profit or commercial advantage and that copies bear this notice and the full citation on the first page. Copyrights for components of this work owned by others than ACM must be honored. Abstracting with credit is permitted. To copy otherwise, or republish, to post on servers or to redistribute to lists, requires prior specific permission and/or a fee. Request permissions from permissions@acm.org.

(c) 2015 ACM 0004-5411/2015/02-ART1 $\$ 15.00$

DOI: http://dx.doi.org/10.1145/2629366 
ConJECTURE 1.1. In polynomial time, one can find a spanning tree of maximum degree at most $k+1$ whose cost is no more than the cost of a minimum cost tree with maximum degree at most $k$.

Note that this conjecture is formulated in the special case where $B_{v}=k$ for each vertex $v \in V$. Goemans [2006] made a major step towards proving this conjecture by giving a polynomial-time algorithm that returns a tree with maximum degree $k+2$, whose cost is at most the cost of a minimum cost tree with maximum degree at most $k$. The algorithm also generalizes when there are different degree bounds on different vertices, where the algorithm returns a spanning tree violating the degree bounds by at most two. In this article, we settle Conjecture 1.1 positively by proving the following result.

THEOREM 1.2. There exists a polynomial-time algorithm for the Minimum Bounded Degree Spanning Tree problem that returns a tree $T$ in which each vertex $v \in V$ has degree at most $B_{v}+1$ and the cost of the tree $T$ is at most OPT, where OPT is the minimum cost of a spanning tree which satisfies all degree bounds.

Theorem 1.2 also generalizes to the setting when there is a degree lower bound $A_{v}$ and a degree upper bound $B_{v}$ for each vertex $v \in V$. In this case, the algorithm returns a spanning tree $T$ such that $A_{v}-1 \leq d_{T}(v) \leq B_{v}+1$ for each vertex $v \in V$ and the cost of $T$ is at most OPT, where OPT is the minimum cost of a spanning tree which satisfies all degree (upper and lower) bounds. Note that we do not assume that the cost function satisfies triangle inequalities (or even non-negativity). With this general cost function, it is not possible to obtain any approximation algorithm if we insist on satisfying all the degree upper bounds [Garey and Johnson 1979]. ${ }^{1}$ Thus, Theorem 1.2 is essentially the best possible.

\subsection{Techniques}

Polyhedral combinatorics has proved to be a powerful, coherent, and unifying tool in combinatorial optimization [Schrijver 2003]. In the last two decades, polyhedral methods have also been applied very successfully to the design of approximation algorithms [Vazirani 2004]. A standard approach to design approximation algorithms is to first formulate the problem as an integer program, and then use the linear relaxation of this program as a way to lower-bound the cost of an optimal solution to a minimization problem. We shall also use this approach. Given an undirected graph $G=(V, E)$ and a subset $S$ of vertices, we use $E(S)=\{e \in E:|e \cap S|=2\}$ to denote the set of edges which have both endpoints in $S \subseteq V$. For any subset of edges $F \subseteq E$ and vertices $S \subseteq V$, we denote $\delta_{F}(S)$ to be the set of edges in $F$ which have exactly one endpoint in $S$. When $F=E$, we drop the subscript $F$ from the notation. For any vertex $v \in V$, we let $\delta_{F}(v)$ denote $\delta_{F}(\{v\})$ and $d_{F}(v)=\left|\delta_{F}(v)\right|$. For $x: E \rightarrow \mathbb{R}^{+}$and $F \subseteq E$, we denote $x(F):=\sum_{e \in F} x(e)$. Following Goemans [2006], we use the following natural linear programming relaxation for the Minimum Bounded Degree Spanning Tree problem.

$$
\begin{array}{rlrl}
\operatorname{minimize} & c(x) & =\sum_{e \in E} c_{e} x_{e} & \\
\text { subject to } & x(E(V)) & =|V|-1 & \\
& x(E(S)) & \leq|S|-1 \quad \forall S \subset V
\end{array}
$$

\footnotetext{
${ }^{1}$ Assuming $\mathrm{P} \neq \mathrm{NP}$, there is no $p(n)$-approximation which satisfies the degree bound exactly for any polynomial $p(n)$ of $n$ where $n$ is the number of vertices.
} 


$$
\begin{aligned}
x(\delta(v)) & \leq B_{v} & & \forall v \in V \\
x_{e} & \geq 0 & & \forall e \in E
\end{aligned}
$$

The constraints (2) state that every tree must have exactly $|V|-1$ edges. Constraints (3) state that for any subset $S \subset V$, the number of edges picked with both endpoints in $S$ must be at most $|S|-1$. These constraints are valid since any spanning tree induces an acyclic subgraph on any subset $S \subseteq V$ and the maximum number of edges in an acyclic subgraph on $S$ is at most $|S|-1$. The constraints (4) state that the number of edges included in the solution is at most $B_{v}$ for each $v \in V$.

Using a polyhedral approach, a general strategy is to construct a spanning tree of cost no more than the optimal value of this linear program, and in which the degree of each vertex $v \in V$ is at most $B_{v}+1$. This would prove Theorem 1.2. In fact, this general strategy has been used in previous work, and different techniques have been proposed to "round" a fractional solution to this linear program. An important observation of Goemans is that an extreme point solution of this linear program is characterized by a laminar family (definitions will be provided later) of tight constraints, inequalities that are satisfied as equalities. This fact was exploited cleverly to obtain the result in Goemans [2006].

We note that a very similar observation was made by Jain [2001] in his breakthrough work on the Survivable Network Design problem, where he first introduced the idea of iterative rounding to the design of approximation algorithms. This potential connection was initiated in Lau et al. [2009], where Jain's iterative rounding method was extended to give the first constant factor (bi-criteria) approximation algorithm for bounded degree network design problems including the Minimum Bounded Degree Steiner Tree problem and the Bounded Degree Survivable Network Design problem. Inspired by these results, we attempted Conjecture 1.1 using the iterative rounding method.

The basic setting of the iterative rounding method for network design problems goes as follows. First, we solve the linear program to obtain an optimal extreme point solution $x^{*}$. We proceed by adding the edges with the highest fractional value to the integral solution. Then we construct the residual problem where the edges added previously are fixed, and update the linear program appropriately. A key feature of the iterative rounding method is to repeat this procedure: solve again the linear program for the residual problem to obtain an optimal extreme point solution (instead of using $x^{*}$ ), and add the edges with the highest fractional value in this new fractional solution to the integral solution. This procedure is iterated until the integral solution constructed is a feasible solution. In the Survivable Network Design problem, the crucial theorem in Jain's approach is that the edges picked in each iteration have fractional value at least $1 / 2$, which ensures that the above algorithm has an approximation ratio of two. This theorem relies heavily on the properties of an extreme point solution, as in the work of Goemans [2006].

Our first contribution is to extend the iterative rounding method and show that it can be used to solve problems optimally. This is achieved by setting integral variables; an variable $e$ such that $x_{e}=0$ is removed and a variable $e$ with $x_{e}=1$ is picked in the solution. One then formulates the residual problem and iterates. The technical claim is to show that one can always find an integral variable in each iteration. We apply this approach to the minimum spanning tree problem and give two different proofs of the integrality of the spanning tree polyhedron in Section 2.

For the Minimum Bounded Degree Spanning Tree problem, however, the direct approach of iterative rounding would not work. The standard iterative rounding framework of picking an edge $e$ with $x_{e}^{*} \geq 1 / 2$ would not work because we can not guarantee the optimality (with respect to the cost of the linear program) of the solution. The latter approach of picking integral edges would not work since we do not expect the linear 
programming solution to only have integral values. Our algorithm uses the iterative relaxation framework as introduced in Lau et al. [2009]. The key insight is that if the algorithm cannot find integral edges to pick, then it can remove/relax one of the degree constraints. In particular, we find a vertex with degree upper bound $B_{v}$ and with at most $B_{v}+1$ edges incident on it in the support of an extreme point solution. The algorithm then removes the degree constraint of $v$ and proceeds to re-solve the linear program. The heart of our analysis is to show that one can always find such a vertex. This is proved by a counting argument which relies heavily on the fact that an extreme point solution is characterized by a laminar family of tight constraints [Jain 2001; Goemans 2006]. Since the algorithm only picks integral edges, the optimality of the cost follows naturally. The condition when the degree constraint of a vertex is removed ensures that the degree constraint can be violated by at most one, giving us Theorem 1.2.

\subsection{Related Work}

The Minimum Bounded Degree Spanning Tree problem is a well-studied problem and has been attacked using a variety of techniques. Initial efforts on the problem were concentrated on obtaining bi-criteria approximation algorithms. Let OPT be the cost of an optimal solution to the MBDST problem. An $\left(\alpha, f\left(B_{v}\right)\right)$-approximation algorithm $^{2}$ is an algorithm which returns a spanning tree $T$ with cost at most $\alpha \cdot$ OPT and $d_{T}(v) \leq f\left(B_{v}\right)$ for all $v$, where $d_{T}(v)$ denotes the degree of $v$ in $T$. Ravi et al. [1993] gave an $\left(O(\log n), O\left(B_{v} \log n\right)\right)$-approximation for the MBDST problem using a matchingbased augmentation technique. Könemann and Ravi [2002, 2005] used a Lagrangianrelaxation based approach to obtain an $\left(O(1), O\left(B_{v}+\log n\right)\right.$ )-approximation algorithm. Chaudhuri et al [2009b, 2009a] presented an $\left(1, O\left(B_{v}+\log n\right)\right)$-approximation algorithm, and an $\left(O(1), O\left(B_{v}\right)\right)$-approximation algorithm based on the push-relabel framework developed for the maximum flow problem. Ravi and Singh [2006] considered a variant of the problem in which the tree returned must be a minimum spanning tree, and gave an algorithm that returns an MST in which the degree of any vertex $v$ is at most $B_{v}+p$, where $p$ is the number of distinct costs in any MST. Recently, Goemans [2006] presented an $\left(1, B_{v}+2\right)$-approximation algorithm using matroid intersection techniques. This was the previous best guarantee for the MBDST problem. In the special case where the graph is unweighted, Fürer and Raghavachari [1994], building on the work of Win [1989], gave an algorithm that returns a spanning tree in which the degree of each vertex $v$ is at most $B_{v}+1$ or returns a witness certifying infeasibility of the degree bounds.

The iterative rounding technique that we use in our algorithm was developed in Jain [2001] for the Survivable Network Design problem and has later been successfully applied to various problems [Cheriyan et al. 2006; Fleischer et al. 2006]. Recently, this technique has been extended to give constant factor bi-criteria approximation algorithm for the Bounded Degree Survivable Network Design problem [Lau et al. 2009; Lau and Singh 2008].

\subsection{Organization}

The rest of this article is organized as follows. In Section 2, we give two iterative algorithms which shows the integrality of the spanning tree polyhedron. Then, in Section 3, we present a new proof of the result of Goemans [2006] that gives an $\left(1, B_{v}+\right.$ 2)-approximation algorithm for the MBDST problem. In Section 4, we present the main algorithm and the proof of Theorem 1.2. Both the results for the MBDST problem build

\footnotetext{
${ }^{2}$ Notice that the first parameter is used to specify the ratio, while the second parameter is used to specify the actual bound.
} 
on the iterative algorithms for the spanning tree problem given in Section 2. Finally, in Section 5, we extend the algorithm to deal with degree lower bounds.

\section{SPANNING TREE POLYHEDRON}

In this section, we present two iterative arguments to show that the minimum spanning tree polytope is integral. This motivates the main result of this article and illustrates the basic proof techniques.

\subsection{Linear Program}

Let $G=(V, E)$ be a graph with a cost function $c$ on edges. A classical result of Edmonds [1971] states that the following linear program LP-MST $(G)$ is integral, and an optimal extreme point solution is always a minimum spanning tree.

$$
\begin{aligned}
& \text { minimize } \quad c(x)=\sum_{e \in E} c_{e} x_{e} \\
& \text { subject to } \quad x(E(V))=|V|-1 \\
& x(E(S)) \leq|S|-1 \quad \forall S \subset V \\
& x_{e} \geq 0 \quad \forall e \in E
\end{aligned}
$$

\subsection{Characterization of the Extreme Point Solutions}

An extreme point solution is defined as the unique solution of $m$ linearly independent tight constraints (constraints which achieve equality), where $m$ denotes the number of variables in the linear program. We focus on extreme point solutions $x^{*}$ such that $x_{e}^{*}>0$ for each $e \in E$. We first give some basic definitions. For a set $F \subseteq E$, we define the characteristic vector of $F$ in $\mathbb{R}^{|E|}, \chi(F)$, as the vector that has a 1 corresponding to each edge $e \in F$, and 0 otherwise. For any set family $\mathcal{F} \subseteq 2^{V}$, let $\operatorname{span}(\mathcal{F})$ be the vector space generated by the set of vectors $\{\chi(E(S)) \mid S \in \mathcal{F}\}$. A family of sets $\mathcal{L} \subseteq 2^{V}$ is laminar if for any two sets in the family, either one contains the other or they are disjoint.

Lemma 2.1 [CoRnuejols ET AL. 1985]. Let $x^{*}$ be any extreme point solution to LP$M S T(G)$ such that $x_{e}^{*}>0$ for each edge $e \in E$ and let $\mathcal{F}=\left\{S \subseteq V\left|x^{*}(E(S))=\right| S \mid-1\right\}$ be the collection of sets corresponding to tight constraints. Then there exists a laminar family $\mathcal{L} \subseteq \mathcal{F}$ such that

(1) The set of vectors $\{\chi(E(S)): S \in \mathcal{L}\}$ are linearly independent and span $(\mathcal{L})=$ $\operatorname{span}(\mathcal{F})$.

(2) $|\mathcal{L}|=|E|$.

(3) $x^{*}$ is the unique solution to the set of equations $\{x(E(S))=|S|-1: S \in \mathcal{L}\}$.

The proof of Lemma 2.1 is quite standard [Cornuéjols et al. 1985], but we include it for completeness. It also illustrates the uncrossing technique.

PRoOF. First, we need the following uncrossing lemma on intersecting sets among tight sets.

Lemma 2.2 [CoRnuejols et AL. 1985]. If $S, T \in \mathcal{F}$ and $S \cap T \neq \varnothing$, then both $S \cap T$ and $S \cup T$ are in $\mathcal{F}$. Furthermore, $\chi(E(S))+\chi(E(T))=\chi(E(S \cap T))+\chi(E(S \cup T))$. 
Proof. As $S \cap T \neq \varnothing$, we have:

$$
\begin{aligned}
|S|-1+|T|-1 & =|S \cap T|-1+|S \cup T|-1 \\
& \geq x^{*}(E(S \cap T))+x^{*}(E(S \cup T)) \\
& \geq x^{*}(E(S))+x^{*}(E(T)) \\
& =|S|-1+|T|-1,
\end{aligned}
$$

where inequality (11) follows from feasibility of constraints for $S \cap T$ and $S \cup T$, inequality (12) follows from supermodularity of the induced edge set function. and hence we have equality throughout. This implies that $S \cup T$ and $S \cap T$ are both in $\mathcal{F}$, and furthermore there are no edges $e \in E^{*}$ between $S \backslash T$ and $T \backslash S$. Therefore, $\chi(E(S))+\chi(E(T))=\chi(E(S \cap T))+\chi(E(S \cup T))$.

Now, we show that any maximal laminar family in $\mathcal{F}$ suffices for the proof of Lemma 2.1.

LEMma 2.3 [CORNUÉJOLS ET AL. 1985; JAIN 2001]. If $\mathcal{L}^{\prime}$ is a maximal laminar subfamily of $\mathcal{F}$, then $\operatorname{span}\left(\mathcal{L}^{\prime}\right)=\operatorname{span}(\mathcal{F})$.

Proof. Let $\mathcal{L}^{\prime}$ be a maximal laminar subfamily of $\mathcal{F}$ and assume that $\chi(E(S)) \notin$ $\operatorname{span}\left(\mathcal{L}^{\prime}\right)$ for some $S \in \mathcal{F}$. Choose one such set $S$ that intersects as few sets of $\mathcal{L}^{\prime}$ as possible. Since $\mathcal{L}^{\prime}$ is a maximal laminar family, there exists $T \in \mathcal{L}^{\prime}$ that intersects $S$. From Lemma 2.2, we have that $S \cap T$ and $S \cup T$ are also in $\mathcal{F}$ and that $\chi(E(S))+$ $\chi(E(T))=\chi(E(S \cap T))+\chi(E(S \cup T))$. Since $\chi(E(S)) \notin \operatorname{span}\left(\mathcal{L}^{\prime}\right)$, either $\chi(E(S \cap T)) \notin$ $\operatorname{span}\left(\mathcal{L}^{\prime}\right)$ or $\chi(E(S \cup T)) \notin \operatorname{span}\left(\mathcal{L}^{\prime}\right)$. In either case, we have a contradiction because both $S \cup T$ and $S \cap T$ intersect fewer sets in $\mathcal{L}^{\prime}$ than $S$; this is because every set in $\mathcal{L}^{\prime}$ that intersects $S \cup T$ or $S \cap T$ must intersect $S$.

A maximal subfamily $\mathcal{L}$ of $\mathcal{L}^{\prime}$ such that $\{\chi(E(S)): S \in \mathcal{L}\}$ is linearly independent suffices to prove property (2.1). And property (2.1) and property (2.1) follow from property (2.1) and the fact that $x^{*}$ is an extreme point solution.

Any laminar family $\mathcal{L}$ defines a directed forest $L$ in which nodes correspond to sets in $\mathcal{L}$ and there exists an edge from set $R$ to set $S$ if $R$ is the smallest set containing $S$. We call $R$ the parent of $S$ and $S$ a child of $R$. For clarity, we will refer to the vertices of forest $L$ by nodes. A node with no parent is called a root and a node with no child is called a leaf. Given a node $R$, the subtree rooted at $R$ consists of $R$ and all its descendants. The following fact is standard and follows easily from induction.

FACT 2.4. Let $\mathcal{L} \subset 2^{V}$ be a laminar family over ground set $V$. Then $|\mathcal{L}| \leq 2|V|-1$ and if each set in $\mathcal{L}$ has size at least two, then $|\mathcal{L}| \leq|V|-1$.

\subsection{Iterative Algorithm}

The iterative MST Algorithm I is given in Figure 1. To prove the correctness of the algorithm, we prove the following two lemmas. First, we prove that the algorithm will terminate.

Lemma 2.5. For any extreme point solution $x^{*}$ to the LP-MST(G) with $x_{e}^{*}>0$ for every edge e, there exists a vertex $v$ with $d(v)=1$.

Proof. Suppose to the contrary that each vertex is of degree at least two. Then $|E|=\frac{1}{2} \sum_{v \in V} d(v) \geq|V|$. On the other hand, since there is no edge $e$ with $x_{e}^{*}=0$, every tight inequality is of the form $x^{*}(E(S))=|S|-1$. By Lemma 2.1, there are $|\mathcal{L}|$ linearly independent tight constraints of the form $x^{*}(E(S))=|S|-1$, where $\mathcal{L}$ is a laminar family with no singleton sets. Moreover, we have $|E|=|\mathcal{L}|$. From Fact 2.4, we have that $|\mathcal{L}| \leq|V|-1$ which is a contradiction. 


\section{Iterative MST Algorithm I}

(1) Initialization $T \leftarrow \varnothing$.

(2) While $V(G) \neq \varnothing$ do

(a) Find an optimal extreme point solution $x^{*}$ of $\operatorname{LP}-\operatorname{MST}(G)$ and remove every edge $e$ with $x_{e}^{*}=0$ from $G$.

(b) Find a vertex $v$ with at most one edge $e=u v$ incident at it, and update $T \leftarrow$ $T \cup\{e\}, G \leftarrow G \backslash\{v\}$.

(3) Return $T$.

Fig. 1. MST Algorithm I.

Next, we show that the returned solution is a minimum spanning tree in the following lemma.

\section{Lemma 2.6. The Iterative MST Algorithm I returns a minimum spanning tree.}

PRoOF. This is proved by induction on the number of iterations of the algorithm. If the algorithm finds a vertex $v$ of degree one (a leaf vertex) in Step 2(b) with an edge $e=\{u, v\}$ incident at $v$, then we must have $x_{e}^{*}=1$ since $x(\delta(v)) \geq 1$ is a valid inequality of the LP (subtracting the constraint $x(E(V-v)) \leq|V|-2$ from the constraint $x(E(V))=|V|-1)$. Observe that the residual problem is to find a minimum spanning tree on $G^{\prime}=G \backslash v$, and the same procedure is applied to solve the residual problem recursively.

Since $x_{e}^{*}=1$, the restriction of $x^{*}$ to $E\left(G^{\prime}\right)$, denoted by $x_{r e s}^{*}$, is a feasible solution to the LP-MST $\left(G^{\prime}\right)$. Therefore, inductively, the algorithm will return a spanning tree $T^{\prime}$ of $G^{\prime}$ of cost at most the optimal value of the $\operatorname{LP}-\operatorname{MST}\left(G^{\prime}\right)$, and hence $c\left(T^{\prime}\right) \leq c \cdot x_{r e s}^{*}$. Therefore,

$$
c(T)=c\left(T^{\prime}\right)+c_{e} \text { and } c\left(T^{\prime}\right) \leq c \cdot x_{r e s}^{*},
$$

which imply that

$$
c(T) \leq c \cdot x_{r e s}^{*}+c_{e}=c \cdot x^{*}
$$

as $x_{e}^{*}=1$. This shows that the algorithm returns a minimum spanning tree of the graph.

Remark 2.7. If $x^{*}$ is an optimal extreme point solution to $\operatorname{LP}-\operatorname{MST}(G)$, then the residual LP solution $x_{\text {res }}^{*}, x$ restricted to $G^{\prime}=G \backslash v$ remains an optimal extreme point solution to LP-MST $\left(G^{\prime}\right)$. Consider the laminar family $\mathcal{L}$ defining $x^{*}$ as given by Lemma 2.1. Due to Lemma 2.3, we can assume, without loss of generality, that $\{u, v\} \in \mathcal{L}$. Now it is easy to see that $\mathcal{L}^{\prime}=\{S \backslash v: S \in \mathcal{L}, S \neq\{u, v\}\}$ defines the solution $x_{\text {res }}^{*}$ by satisfying all conditions of Lemma 2.1 and thus witnessing that $x_{r e s}^{*}$ is an extreme point of LP$\operatorname{MST}\left(G^{\prime}\right)$. Hence, in the iterative MST algorithm I, we only need to solve the original linear program once and none of the residual linear programs.

We give another algorithm which achieves the same guarantee as the previous algorithm but is even simpler (see Figure 2). Instead of finding integral edges one by one, we can directly prove that all the variables must be integral by a similar counting argument.

\section{Lemma 2.8. Iterative MST Algorithm II returns a minimum spanning tree of $G$.}

Proof. We first claim that $|T|=|V|-1$ where $T$ is the set of edges returned by the algorithm. Since $T$ is the support of $x^{*}$, and we have $x^{*}(E)=|V|-1$ and $x_{e}^{*} \leq 1$ for each edge $e \in E$, we must have $|T| \geq|V|-1$. Moreover, since $x^{*}$ is extreme, we must have that the number of nonzero edges $|T|$ equals the size of a laminar family $\mathcal{L}$ defining 


\section{Iterative MST Algorithm II}

(1) Find an optimal extreme point solution $x^{*}$ to the $\operatorname{LP}-\mathrm{MST}(G)$ and remove every edge $e$ with $x_{e}^{*}=0$ from $E$.

(2) Return $T=\left\{e: x_{e}^{*}>0\right\}$.

Fig. 2. Iterative MST Algorithm II.

$x^{*}$ as given by Lemma 2.1. Since, $|\mathcal{L}| \leq|V|-1$ we have $|T| \leq|V|-1$ giving us the equality.

Then, we also have that $x_{e}^{*}=1$ for each edge $e \in T$. Thus, $x^{*}$ is an integral feasible solution to $\operatorname{LP}-\operatorname{MST}(G)$ and therefore $T$ is a minimum spanning tree.

\section{A +2 APPROXIMATION ALGORITHM}

In this section, we prove the following theorem using the iterative relaxation technique.

Theorem 3.1 [Goemans 2006]. There exists a polynomial time algorithm for the Minimum Bounded Degree Spanning Tree problem that returns a spanning tree T such that $c(T) \leq$ opt and $d_{T}(v) \leq B_{v}+2$ for each vertex $v \in V$, where opt is the cost of the optimal solution satisfying all degree bounds exactly.

\subsection{Linear Programming Relaxation}

We use the following standard linear programming relaxation for the MBDST problem, which we denote by $\operatorname{LP}-\operatorname{MBDST}(G, W)$. In the following, we assume that degree bounds are given for vertices only in a subset $W \subseteq V$.

$$
\begin{aligned}
\operatorname{minimize} & c(x) & =\sum_{e \in E} c_{e} x_{e} & \\
\text { subject to } & x(E(V)) & =|V|-1 & \\
x(E(S)) & \leq|S|-1 & & \forall S \subset V \\
x(\delta(v)) & \leq B_{v} & & \forall v \in W \\
x_{e} & \geq 0 & & \forall e \in E
\end{aligned}
$$

Observe that LP-MBDST $(G, W)$ has an exponential number of constraints. Cunningham [1984] gave a polynomial-time procedure to separate over constraints (15)-(16) and (18) and it can be reduced to $2|V|-2$ min-cut computations (See Lau et al. [2011] Chapter 4, for details). Separating over constraints (17) is clearly in polynomial time. Hence, using the ellipsoid algorithm [Grötschel et al. 1981], one can optimize over $\operatorname{LP}-\operatorname{MBDST}(G, W)$ in polynomial time.

\subsection{Characterization of Extreme Point Solutions}

We show the following lemma which characterizes any extreme point solution with a family of tight constraints.

LEMMA 3.2. Let $x^{*}$ be an extreme point solution of $\operatorname{LP}-\operatorname{MBDST}(G, W)$ with support $E^{*}$. Then there exists a set $X \subseteq W$ and a laminar family $\mathcal{L}$ such that $x^{*}$ is the unique solution to the following linear system.

$$
\begin{cases}x^{*}(\delta(v))=B_{v} & \forall v \in X \\ x^{*}(E(S))=|S|-1 & \forall S \in \mathcal{L}\end{cases}
$$




\section{MBDST Algorithm I}

(1) Initialization $T \leftarrow \varnothing, W \leftarrow V$.

(2) While $V(G) \neq \varnothing$ do

(a) Find an optimal extreme point solution $x^{*}$ to $\operatorname{LP}-\operatorname{MBDST}(G, W)$ and remove every edge $e$ with $x_{e}^{*}=0$ from $G$. Let the support of $x^{*}$ be $E^{*}$.

(b) If there exists a vertex $v \in V$ with at most one edge $e=u v$ incident at $v$ in $E^{*}$, then update $T \leftarrow T \cup\{e\}, G \leftarrow G \backslash\{v\}, W \leftarrow W \backslash\{v\}$, and $B_{u} \leftarrow B_{u}-1$.

(c) If there exists a vertex $v \in W$ with $d_{E^{*}}(v) \leq 3$, then update $W \leftarrow W \backslash\{v\}$.

(3) Return $T$.

Fig. 3. MBDST Algorithm I.

Moreover, the characteristic vectors $\{\chi(E(S)): S \in \mathcal{L}\} \cup\{\chi(\delta(v)): v \in X\}$ are linearly independent. Furthermore, $\left|E^{*}\right|=|\mathcal{L}|+|X|$.

Proof. The proof follows the same lines as the proof of Lemma 2.1. Let $\tau=\{S \subseteq$ $\left.V: x^{*}(E(S))=|S|-1\right\}$ be the set of all tight constraints from (15) and (16). Following the same uncrossing arguments as in Lemma 2.1, we obtain that there is a laminar family $\mathcal{L} \subseteq \tau$ such that $\operatorname{span}(\tau)=\operatorname{span}(\mathcal{L})$ and constraints corresponding to sets in $\mathcal{L}$ are linearly independent. Let $X$ be a maximal set of vertices such that $x^{*}(\delta(v))=B_{v}$ for each $v \in X$ and the constraints in $X$ are linearly independent with constraints in $\mathcal{L}$. By construction, the constraints corresponding to sets in $\mathcal{L}$ and vertices in $\tau$ are maximally linearly independent and therefore $\left|E^{*}\right|=|\mathcal{L}|+|X|$.

\subsection{Iterative Algorithm}

Our algorithm proving Theorem 3.1 is given in Figure 3. It is a simple iterative algorithm and an extension of Iterative MST Algorithm I. The main addition is Step 2(c) that iteratively relaxes degree constraints.

The correctness of the algorithm follows from the following two lemmas.

Lemma 3.3. Suppose that the MBDST Algorithm I returns T in Step (3). Then T is a spanning tree of cost at most $c\left(x^{*}\right)$ where $x^{*}$ is the optimal LP solution to LP$\operatorname{MBDST}(G, V)$, and the degree of vertex $v$ in $T$ is at most $B_{v}+2$ for each vertex $v \in V$.

Proof. Assuming that the algorithm returns a solution $T$ in Step (3), then the fact that $T$ is a spanning tree of cost at most $c\left(x^{*}\right)$ follows from an argument identical to that in the proof of Lemma 2.6. The degree bound follows simply as well. While a vertex is in $W$, the degree constraint is satisfied exactly by the current fractional solution. If a vertex $v$ is removed from $W$ in Step 2(c), it has only three edges incident at it and the residual degree bound $B_{v}$ is at least one. Thus, even if the algorithm picks all three edges incident at $v$, the degree bound is violated by at most two.

In the next lemma, we prove that in each iteration we can proceed by applying either Step 2(b) or Step 2(c); this will ensure that the algorithm terminates.

Lemma 3.4. Any extreme point solution $x^{*}$ to $\operatorname{LP}-\operatorname{MBDST}(G, W)$ with support $E^{*}$ must satisfy one of the following.

(a) There is a vertex $v \in V$ with $d_{E^{*}}(v)=1$.

(b) There is a vertex $v \in W$ with $d_{E^{*}}(v) \leq 3$.

PRoOF. Suppose for a contradiction that both (a) and (b) are not satisfied. Then every vertex has at least two edges incident on it and every vertex in $W$ has at least four edges incident on it. Therefore, $\left|E^{*}\right| \geq(2(n-|W|)+4|W|) / 2=n+|W|$, where $n=|V(G)|$. 


\section{MBDST Algorithm II}

(1) Let $W \leftarrow V$.

(2) While $W \neq \varnothing$ do

(a) Find an optimal extreme point solution $x^{*}$ to $\operatorname{LP}-\operatorname{MBDST}(G, W)$ and remove every edge $e$ with $x_{e}^{*}=0$ from $G$. Let the support of $x^{*}$ be $E^{*}$.

(b) If there exists a vertex $v \in W$ such that $d_{E^{*}}(v) \leq B_{v}+1$ then update $W \leftarrow$ $W \backslash\{v\}$.

(3) Find an optimal extreme point solution $x^{*}$ of $\operatorname{LP}-\operatorname{MBDST}(G, \varnothing)$ and remove every edge $e$ with $x_{e}^{*}=0$ from $G$.

(4) Return $T$, the support of $x^{*}$.

Fig. 4. MBDST Algorithm II.

By Lemma 3.2, there is a laminar family $\mathcal{L}$ and a set $X \subseteq W$ of vertices such that $\left|E^{*}\right|=|\mathcal{L}|+|X|$. As $\mathcal{L}$ contains subsets of size at least two, $|\mathcal{L}| \leq|V|-1$. Hence, $\left|E^{*}\right|=|\mathcal{L}|+|X| \leq|V|-1+|X| \leq|V|-1+|W|$, a contradiction.

In each iteration, we either remove a degree constraint or include an edge in our solution. Therefore, in a total of at most $|V|+|V|-1=2|V|-1$ iterations, we construct a spanning tree. These steps provide a $\left(1, B_{v}+2\right)$-approximation algorithm for the MBDST problem.

\section{A +1 APPROXIMATION ALGORITHM}

In this section, we give an algorithm that proves Theorem 1.2. The algorithm is an extension of the Iterative MST Algorithm II and is given in Figure 4. In each step of the algorithm, we find an appropriately chosen vertex whose degree constraint is present and remove the degree constraint. Finally, when all the degree constraints are removed, we return the support of the linear programming solution. This is guaranteed to be a spanning tree following from the analysis of Iterative MST Algorithm II in Lemma 2.8.

We argue the correctness of the algorithm in the following two lemmas. In the first part, we assume that the algorithm does terminate and returns the set $T$ when $W=\varnothing$, and show that the solution returned satisfies the claimed guarantee. In the next lemma, we show that in each iteration, the algorithm finds a vertex $v \in W$ satisfying the condition in Step 2(b).

Lemma 4.1. Suppose the MBDST Algorithm II returns $T$ in Step (4), then T is a spanning tree of cost at most $c\left(x^{*}\right)$ where $x^{*}$ is the optimal LP solution to LP-MBDST $(G, V)$ and degree of vertex $v$ in $T$ is at most $B_{v}+1$ for each vertex $v \in V$.

Proof. First, we show that $T$ is a tree. At the end of the last iteration, let $G^{\prime}$ denote the current graph where edges with fractional value zero have been removed in previous iterations. The algorithm then solves $\operatorname{LP}-\operatorname{MBDST}\left(G^{\prime}, \varnothing\right)$ which is exactly the same as linear program LP-MST $\left(G^{\prime}\right)$. Thus, from Lemma 2.8, the optimal solution to the linear program is integral and $T$ is a spanning tree. It is straightforward to check that the optimal linear programming solution of any iteration remains feasible for the next iteration, since we either remove a constraint or remove edges which are set to zero by the fractional solution. Thus the cost of the optimal solution in next iteration does not increase. Therefore, the cost of $T$ is at most the cost of the optimal solution to the initial linear program LP-MBDST $(G, W)$.

Next, we argue that the degree of each vertex $v \in V$ in the returned solution $T$ is at most $B_{v}+1$. Let $v$ be any vertex and consider the iteration when the degree constraint of $v$ is removed. Let $E^{*}$ be the support of the linear programming solution in that 
iteration. We have $d_{E^{*}}(v) \leq B_{v}+1$ and in any further iteration we only remove edges incident at this vertex and never add any other edge. Thus, the final solution $T \subseteq E^{*}$ and therefore $d_{T}(v) \leq B_{v}+1$ as claimed.

In the next lemma, we show that the algorithm does perform correctly and returns a solution. We describe a simple proof due to Bansal et al. [2008].

LEMMA 4.2. Let $x^{*}$ be an extreme point solution to $\operatorname{LP}-\operatorname{MBDST}(G, W)$ and $E^{*}=\{e$ : $\left.x_{e}^{*}>0\right\}$. If $W \neq \varnothing$, then there exists a $v \in W$ such that $d_{E^{*}}(v) \leq B_{v}+1$.

Proof. Let $X \subseteq W$ be the tight degree constraints and $\mathcal{L}$ be laminar family as given by Lemma 2.1. If $X=\varnothing$, then Lemma 2.8 implies that $E^{*}$ is a tree and $x_{e}^{*}=1$ for each $e \in E^{*}$. Thus, $d_{E^{*}}(v)=x^{*}(\delta(v)) \leq B_{v}$ for each $v \in W$ and we are done. In the rest we assume that $X \neq \varnothing$.

Suppose to the contrary that $d_{E^{*}}(v) \geq B_{v}+2$ for each $v \in W$. We now show a contradiction by a counting argument. We give one token to each edge in $E^{*}$. We then redistribute the token such that each vertex in $X$ and each set in $\mathcal{L}$ gets one token and we still have extra tokens left. This will contradict $\left|E^{*}\right|=|X|+|\mathcal{L}|$. The token redistribution is as follows. Each edge $e \in E^{*}$ gives $x_{e}^{*}$ fractional token to the smallest set in $\mathcal{L}$ containing both endpoints of $e$, and $\left(1-x_{e}^{*}\right) / 2$ fractional token to each of its endpoints for the degree constraints, if present.

We show that each set $S \in \mathcal{L}$ obtains one token. $S$ receives $x_{e}^{*}$ token for each edge $e$ such that $S$ is the smallest set containing both endpoints of $e$. Let $R_{1}, \ldots, R_{k}$ be the children of $S$ in the laminar family $\mathcal{L}$ where $k \geq 0$. We have

$$
\begin{aligned}
x^{*}(E(S)) & =|S|-1, \text { and } \\
x^{*}\left(E\left(R_{i}\right)\right) & =\left|R_{i}\right|-1 \text { for each } 1 \leq i \leq k \\
\Longrightarrow x^{*}(E(S))-\sum_{i=1}^{k} x^{*}\left(E\left(R_{i}\right)\right) & =|S|-1-\sum_{i=1}^{k}\left(|R|_{i}-1\right) \\
\Longrightarrow x^{*}(A) & =|S|-1-\sum_{i=1}^{k}\left(\left|R_{i}\right|-1\right),
\end{aligned}
$$

where $A=E(S) \backslash\left(\cup_{i=1}^{k} E\left(R_{i}\right)\right)$. Observe that $S$ receives $x_{e}^{*}$ tokens for each edge $e \in A$ for a total of $x^{*}(A)$ tokens which is an integer by this equation. If $x^{*}(A)=0$, then $A=\varnothing$ and therefore, $\chi(E(S))=\sum_{i=1}^{k} \chi\left(E\left(R_{i}\right)\right)$ which contradicts the linear independence of the constraints in $\mathcal{L}$. Hence, each set $S$ receives at least one token.

Now, we show that each vertex with a tight degree constraint gets one token. Let $v \in X$ be such a vertex. Then $v$ receives $\left(1-x_{e}^{*}\right) / 2$ token for each edge incident at $v$ for a total token of value

$$
\sum_{e \in \delta_{E^{*}}(v)} \frac{1-x_{e}^{*}}{2}=\frac{d_{E^{*}}(v)-B_{v}}{2} \geq 1,
$$

where the first equality holds since $\sum_{e \in \delta_{E^{*}}(v)} x_{e}^{*}=B_{v}$ and the inequality holds since $d_{E^{*}}(v) \geq B_{v}+2$ for each $v \in X$.

To finish the proof, we argue that there is some extra token left for contradiction. If $V \notin \mathcal{L}$, then there exists an edge $e$ which is not contained in any set of $\mathcal{L}$ and the $x_{e}^{*}$ token for that edge gives us the contradiction. Similarly, if there is a vertex $v \in W \backslash X$, then $v$ also collects one token which it does not need and we get the desired contradiction. Moreover, if there is a vertex $v \in V \backslash X$, then each edge $e$ incident at $v$ must have $x_{e}^{*}=1$; otherwise the $\left(1-x_{e}^{*}\right) / 2>0$ token is extra. Note that $\chi(e) \in \operatorname{span}(\mathcal{L})$ for each $e$ with 
$x_{e}^{*}=1$, since $e$ is a tight set of size two. We have

$$
2 \chi(E(V))=\sum_{v \in V} \chi(\delta(v))=\sum_{v \in X} \chi(\delta(v))+\sum_{v \in V-X} \chi(\delta(v))=\sum_{v \in X} \chi(\delta(v))+\sum_{v \in V-X} \sum_{e \in \delta(v)} \chi(e) .
$$

We have argued that $V \in \mathcal{L}$ and $\chi(e) \in \operatorname{span}(\mathcal{L})$ for each edge $e \in \delta(v)$ for $v \in V-X$. Since $X \neq \varnothing$, this implies the linear dependence of the tight constraints in $X$ and those in $\mathcal{L}$, giving us the contradiction.

\section{UPPER AND LOWER DEGREE BOUNDS}

In this section, we consider an extension of the MBDST problem in which a degree lower bound $A_{v}$ and a degree upper bound $B_{v}$ are given for each vertex $v$, and the goal is to find a tree of minimum cost satisfying both upper and lower degree bounds. We prove the following theorem.

THeORem 5.1. There is a polynomial-time algorithm for the Minimum Bounded Degree Spanning Tree problem with upper and lower degree constraints which returns a tree $T$ such that $A_{v}-1 \leq d_{T}(v) \leq B_{v}+1$ for each $v \in V$ and $c(T) \leq o p t$, where opt is the cost of the optimal tree satisfying degree bounds exactly.

The proof of Theorem 5.1 has a similar structure as the proof of Theorem 1.2. Since both upper and lower degree bounds are present, the step of relaxing the degree constraint is more restrictive. Thus, the proof of Lemma 5.3, showing that the algorithm makes progress in each step, becomes involved.

\subsection{Linear Programming Relaxation}

We assume the degree bounds are given on a subset of vertices $W \subseteq V$. The following is a linear programming relaxation for the MBDST problem, which is denoted by LP$\operatorname{MBDST}(G, W, F)$. The edge set $F$ denotes the set of edges which the linear program has assigned a value of one in a previous iteration. We maintain that in all further iterations edges in $F$ will be chosen integrally. Inductively, we will also maintain that edges in $F$ form a forest.

$$
\begin{aligned}
\operatorname{minimize} \quad c(x) & =\sum_{e \in E} c_{e} x_{e} & & \\
\text { subject to } \quad x(E(V)) & =|V|-1 & & \\
x(E(S)) & \leq|S|-1 \quad \forall S \subseteq V & & \\
x(\delta(v)) & \geq A_{v} & & \forall v \in W \\
x(\delta(v)) & \leq B_{v} & & \forall v \in W \\
x_{e} & =1 & & \forall e \in F \\
x_{e} & \geq 0 & & \forall e \in E
\end{aligned}
$$

\subsection{Characterization of Extreme Point Solutions}

We now give a characterization of the extreme point solutions of $\operatorname{LP}-\operatorname{MBDST}(G, W, F)$. Let $\mathcal{C}$ denote the set of vertex sets of the nontrivial connected components spanned by edges in $F$. The proof of the following lemma is similar to the proof of Lemma 3.2.

Lemma 5.2. Let $x^{*}$ be any extreme point solution to $\operatorname{LP}-M B D S T(G, W, F)$. Then there exists a set $X_{L} \subseteq W$, a set $X_{U} \subseteq W$ and a laminar family $\mathcal{L}$ such that $x^{*}$ is the unique 


\section{MBDST Algorithm III}

(1) Initialize $F \leftarrow \varnothing, W \leftarrow V$.

(2) While $F$ is not a spanning tree do

(a) Find an optimal extreme point solution $x^{*}$ to $\operatorname{LP}-\operatorname{MBDST}(G, W, F)$ and remove every edge $e$ with $x_{e}^{*}=0$ from $G$.

(b) If there exists an edge $e=\{u, v\}$ such that $x_{e}^{*}=1$, then $F \leftarrow F \cup\{e\}$.

(c) If there exists a vertex $v \in W$ such that there are at most two edges incident at $v$ with $0<x_{e}^{*}<1$, then update $W \leftarrow W \backslash\{v\}$.

(3) Return $F$.

Fig. 5. MBDST Algorithm III.

solution to the following linear system.

$$
\begin{cases}x^{*}(\delta(v))=A_{v} & \forall v \in X_{L} \\ x^{*}(\delta(v))=B_{v} & \forall v \in X_{U} \\ x^{*}(E(S))=|S|-1 & \forall S \in \mathcal{L}\end{cases}
$$

Moreover,

(1) the vectors $\{\chi(E(S)): S \in \mathcal{L}\} \cup\left\{\chi(\delta(v)): v \in X_{L}\right\} \cup\left\{\chi(\delta(v)): v \in X_{U}\right\}$ are linearly independent.

(2) $\left|E^{*}\right|=|\mathcal{L}|+\left|X_{L}\right|+\left|X_{U}\right|$.

(3) Then $\mathcal{C} \subseteq \mathcal{L}$.

Proof. The proof follows along the same lines as in the proofs of Lemma 2.1 and Lemma 3.2. Let $\tau=\left\{S \subseteq V: x^{*}(E(S))=|S|-1\right\}$ be the set of all tight spanning tree constraints in LP-MBDST $(G, W, F)$. By uncrossing arguments, we can choose any maximal laminar $\mathcal{L}$ such that the constraints for sets in $\mathcal{L}$ are linearly independent to ensure that $\operatorname{span}(\mathcal{L})=\operatorname{span}(\tau)$. Let $S \in C$ be a nontrivial connected component spanned by edges in $F$. Then we have $x^{*}(E(S)) \geq|F \cap \delta(S)|=|S|-1$. But feasibility of $x^{*}$ implies that $x^{*}(E(S)) \leq|S|-1$ and therefore the constraints for set $S$ must be tight. Moreover, every edge with nonzero fractional value with both endpoints in $S$ must be in $F$. It is easy to see that the constraints for sets $S \in \mathcal{C}$ are all linearly independent since they involve disjoint set of edges. From Lemma 2.3, it follows that we can choose a laminar family $\mathcal{L}$ such that $\mathcal{C} \subseteq \mathcal{L}$. The rest of the conditions follow simply by choosing a maximal collection of vertices with tight degree constraints $X_{L}$ and $X_{U}$ while ensuring linear independence.

\subsection{Iterative Algorithm}

The algorithm is given in Figure 5. In each iteration, we either pick an integral edge or remove one of the degree constraints. Due to presence of upper and lower degree constraints, we only remove degree constraints on vertices which have at most two strictly fractional edges incident at them.

For the correctness of the MBDST Algorithm III, we shall prove the following key lemma, which will ensure that the algorithm terminates.

Lemma 5.3. An extreme point solution $x^{*}$ of $\operatorname{LP}-M B D S T(G, W, F)$ with support $E^{*}$ must satisfy one of the following.

(a) There is an edge $e \in E^{*} \backslash F$ such that $x_{e}^{*}=1$ or $x_{e}^{*}=0$.

(b) There is a vertex $v \in W$ such that there at most two edges incident at $v$ with fractional value strictly between 0 and 1 .

Before we prove Lemma 5.3, we use it to prove Theorem 5.1. 
Proof OF Theorem 5.1. Lemma 5.3 ensures that in each iteration we either remove a degree constraint or reduce the number of strictly fractional edges. It is also straightforward to see that the same linear programming solution remains an extreme point solution after we apply Step 2(a) or $2(\mathrm{~b})$, and we need to resolve the linear program only after we apply Step 2(c). Hence, the algorithm will terminate in at most $|V|$ iterations. Moreover, the linear programming solution at any iteration is feasible to the linear program solved in the next iteration. Thus, the cost of the optimal solution to the linear programming formulation can only decrease. Hence, the cost of the final solution returned is at most the cost of the optimal fractional solution to the initial linear program, which is a lower bound on the optimum.

Consider any vertex $v \in V$. We argue that both upper-and lower-degree constraint are satisfied within an additive error of one. Consider the iteration when the constraint for vertex $v$ is removed. Let $x^{*}$ denote the optimal fractional solution obtained in this iteration, $F^{\prime}=\left\{e: x_{e}^{*}=1\right\}$ denote the set of edges with fractional value one, and $E^{*}=\left\{e: x_{e}^{*}>0\right\}$ denote the support of $x^{*}$. Since the constraint for vertex $v$ is removed in this iteration, we have that $d_{E^{*} \backslash F^{\prime}}(v) \leq 2$. Since every edge in $F^{\prime}$ incident at $v$ will be present in the final tree $F$ returned by the algorithm, $d_{F}(v) \geq d_{F^{\prime}}(v) \geq\left\lfloor x^{*}\left(\delta_{E^{*}}(v)\right)\right\rfloor-1 \geq$ $A_{v}-1$, where we use the fact that $\delta_{E^{*}}(v)$ has at most two fractional edges. Similarly, $F$ can contain at most two more edges incident at $v$ that are not included in $F^{\prime}$. Thus, $d_{F}(v) \leq d_{F^{\prime}}(v)+2 \leq\left\lceil x^{*}\left(\delta_{E^{*}}(v)\right)\right\rceil+1 \leq B_{v}+1$.

It remains to complete the proof of Lemma 5.3.

Proof of Lemma 5.3. Let $\mathcal{L}$ be the laminar family and $X:=X_{L} \cup X_{U}$ be the vertices defining the solution $x^{*}$ as in Lemma 5.2. Suppose that both (a) and (b) of Lemma 5.3 are not satisfied. We shall derive that $|\mathcal{L}|+|X|<\left|E^{*}\right|$, which will contradict Lemma 5.2 and complete the proof. We also let $E^{\prime}=E^{*} \backslash F$ be the set of edges which are fractional in the linear programming solution $x^{*}$.

As before, we do this by a counting argument. We first assign two tokens for each edge $e$ for a total of $2\left|E^{*}\right|$ tokens. In the first assignment, if $e \in E^{\prime}$, that is, $x_{u v}^{*}<1$, it gives one token to each of its endpoints $u$ and $v$. If $e \in F$, that is, $x_{u v}^{*}=1$, it gives both of its tokens to the smallest set in $\mathcal{L}$ containing both $u$ and $v$.

Let $S \in \mathcal{C}$ be the vertex set of any nontrivial connected component of $F$. We first show that the tokens from edges in $F$ are enough to give two tokens to each set in $R \in \mathcal{L}$ such that $R \subseteq S$.

ClaIm 5.4. The tokens assigned by edges in $F$ are enough to give two tokens to each member $R \in \mathcal{L}$ such that $R \subseteq S$ for some $S \in \mathcal{C}$.

Proof. Observe that every edge $e$ with both endpoints in $R$ must be in $F$ and therefore $x_{e}^{*}=1$. Let $R_{1}, \ldots, R_{k}$ be the children of $R$ in forest $L$. Then, we have $E^{*}(R) \backslash \cup_{i} E^{*}\left(R_{i}\right) \neq \varnothing$, since the constraint for set $R$ is independent of the constraints for set $R_{i}$ 's. So, any edge $e \in E^{*}(R) \backslash \cup_{i} E^{*}\left(R_{i}\right)$ gives two tokens to $R$ as claimed.

We now argue that enough tokens are assigned to rest of the sets in $\mathcal{L}$ and vertices in $X$ by a careful inductive argument. We first construct a new laminar family of the remaining sets. We remove all sets from $\mathcal{L}$ that are strictly contained in some $S \in \mathcal{C}$. We also add all singleton sets $\{v\}$ that are not contained in any $S \in \mathcal{C}$ to the laminar family. We call the new laminar family $\mathcal{L}^{\prime}$ and $L^{\prime}$ be the forest associated with laminar family $\mathcal{L}^{\prime}$. By construction, leaves of $\mathcal{L}^{\prime}$ are either sets of size one or sets in $\mathcal{C}$ and form a partition of $V$. Similarly, for any node $S$, the set of children of $S$ forms a partition of $S$.

Observe that sets in $\mathcal{C}$ do not need to be assigned any new tokens since edges in $F$ assign them two token by Claim 5.4. Similarly sets of size one, $\{v\}$ where $v \notin X$ do 
not need any tokens. But we include them in $\mathcal{L}^{\prime}$ for induction. Let $Y$ denote the set of vertices which have an edge in $E^{\prime}$ incident on them. We call the vertices in $Y$ active. Observe that in the initial assignment each vertex in $X$ gets at least three tokens since it has at least three edges from $E^{\prime}$ incident on it. Since we need to assign only two tokens to the degree constraint, it has at least one extra token. An active vertex not in $X$ does not need any token. Thus, every active vertex has at least one extra token. These extra tokens will be carefully reassigned to sets in $\mathcal{L}^{\prime}$.

The following definition gives a characterization of those sets which need a careful analysis.

Definition 5.5. A set $S \in \mathcal{L}^{\prime}$ such that $S \neq V$ is special if:

(1) $\left|\delta_{E^{\prime}}(S)\right|=3$;

(2) $x^{*}\left(\delta_{E^{\prime}}(S)\right)=1$ or $x^{*}\left(\delta_{E^{\prime}}(S)\right)=2$;

(3) $\chi\left(\delta_{E^{\prime}}(S)\right)$ is a linear combination of the characteristic vectors of its descendants (including possibly $\chi\left(E^{\prime}(S)\right)$ ) and the characteristic vectors $\chi\left(\delta_{E^{\prime}}(v)\right)$ of $v \in S \cap X$.

From now on, to reduce notation, we drop the subscript $E^{\prime}$ in $d_{E^{\prime}}$ and $\delta_{E^{\prime}}$.

Claim 5.6. A leaf in $\mathcal{L}^{\prime}$ is special if and only if it is in one of the following two cases:

(1) $S \in \mathcal{C}$ and contains exactly one active vertex $v$ where $v \in X$ and $|\delta(v)|=3$, or

(2) $S=\{v\}$ where $v \in X$ and $|\delta(v)|=3$.

Proof. Consider a special leaf $S$ where $S \in \mathcal{C}$ or $S=\{v\}$. Then, $\delta(S)=\cup_{u \in S \cap Y} \delta(u)$ and $\delta(u) \cap \delta(w)=\varnothing$ since there is no edge in $E^{\prime}$ with both endpoints $\{u, w\} \in S$. From Property 3 of a special set, it follows that each $v \in Y \cap S$ must be in $X$. Since $|\delta(S)|=3$ and $|\delta(v)| \geq 3$ for each $v \in X$, we have that $\delta(S)=\delta(v)$ for some $v \in X \cap S$ and $|\delta(v)|=3$ as required. Therefore, if $S \in \mathcal{C}$, there is exactly one active vertex $v \in S$ and $v \in X$ and $|\delta(v)|=3$. Similarly, if $S=\{v\}$, then $v \in X$ and $|\delta(v)|=3$.

For the other direction, every leaf which contains exactly one active vertex where $v \in X$ with $|\delta(S)|=3$ satisfies the first condition. The second condition is satisfied since each of the tree edges incident at it are strictly fractional and therefore $0<x^{*}(\delta(v))<3$. Since, $v \in X, x^{*}(\delta(v))$ is an integer and therefore must equal 1 or 2 satisfying the second condition. The third condition is straightforward since $\chi(\delta(S))=\chi(\delta(v))$.

The following lemma will complete the proof of Lemma 5.3, and hence Theorem 5.1. The lemma says that inductively we can assign required number of token to each set in $\mathcal{L}^{\prime}$, and at least one extra token to the root. Of course, the required number of tokens is zero for each leaf $S \in \mathcal{C}$ and $S=\{v\}$ where $v \notin X$. The rest of the nodes in $L^{\prime}$ require at least two tokens. Moreover, the root will get exactly one extra token only if it is special.

LEMMA 5.7. For any rooted subtree of the forest $L^{\prime} \neq \varnothing$ with root $S$, we can distribute the tokens assigned to vertices inside $S$ such that every vertex in $X \cap S$ gets at least two tokens. Moreover, every node in the subtree which is not in $\mathcal{C}$ or $\{v\}$ where $v \notin X$ gets at least two tokens. Finally, the root gets at least one extra token and exactly one only if $S$ is special or $S=V$.

Proof. First, we prove a claim needed for the lemma.

Claim 5.8. If $S \neq V$ and $S \in \mathcal{L}^{\prime}$, then $|\delta(S)| \geq 2$.

Proof. Since $S \neq V, x^{*}(\delta(S)) \geq 1$ is a valid inequality of the LP. Since $S \in \mathcal{L}^{\prime}$ and is not strictly contained in the vertex set of a component spanned by $F$, we have that $F \cap \delta(S)=\varnothing$. Thus, $x_{e}^{*}<1$ for each $e \in \delta(S)$ and $|\delta(S)| \geq 2$.

Base Case. Consider a leaf $S$ of tree $L^{\prime}$. First, consider the case when $S=\{v\}$. From Claim 5.6, if $v \in X$, we have $d(v) \geq 3$ and exactly three only if $\{v\}$ is special. In the 
initial assignment, $v$ is assigned $d(v)$ tokens. Since $v$ needs to be assigned two tokens, it has at least one extra token and exactly one extra token only if $S=\{v\}$ is special. If $v \notin X$, then $v$ receives $d(v) \geq 2$ tokens from Claim 5.8. Since it does not need any tokens, both tokens are extra tokens.

Next, consider the case when $S \in \mathcal{C}$. Then again each active vertex in $S \cap Y$ receives at least one extra token. If $S$ contains two active vertices, we have the two extra tokens to assign to $S$. Recall that $S$ itself does not need any tokens since it has been assigned two tokens from edges in $F$. Otherwise, consider the case when $S$ contains exactly one active vertex, say $v$. If $v \notin X$, then $|\delta(S)|=|\delta(v)| \geq 2$ from Claim 5.8 and we have the two extra tokens. If $v \in X$, then $|\delta(v)|=3$ if and only if $S$ is special from Claim 5.6 and $|\delta(v)| \geq 4$ otherwise. Since $v$ needs to be assigned two tokens, we can assign one extra token to $S$ if $S$ is special and at least two otherwise.

Induction Step. The following two claims are useful in the induction step. Recall that $E^{*}(S)$ denotes the set of edges with both endpoints in $S$. We denote by $D(S)$ the set of edges with endpoints in different children of $S$ in the forest $L^{\prime}$. Observe that if $S$ is not a leaf in $\mathcal{L}^{\prime}$, then $D(S) \subseteq E^{\prime}$, that is, all edges in $D(S)$ are strictly fractional.

Claim 5.9. If $S \in \mathcal{L}^{\prime}$ has $r \geq 1$ children, then $x^{*}(D(S))=r-1$.

Proof. Let the children of $S$ be $R_{i}, 1 \leq i \leq r$. We have

$$
x^{*}\left(E^{*}\left(R_{i}\right)\right)=\left|R_{i}\right|-1 .
$$

Observe that this equality also holds for children $R_{i}$ where $\left|R_{i}\right|=1$. In this case, $E^{*}\left(R_{i}\right)=\varnothing$ and both the left-hand side and right-hand side are zero. As $S \in \mathcal{L}$, we have

$$
x^{*}\left(E^{*}(S)\right)=|S|-1
$$

Since $S=\bullet_{i} R_{i}$, we obtain

$$
\begin{aligned}
x^{*}(D(S)) & =x^{*}\left(E^{*}(S)\right)-\sum_{i} x^{*}\left(E^{*}\left(R_{i}\right)\right) \\
& =|S|-1-\sum_{i}\left(\left|R_{i}\right|-1\right) \\
& =\left(|S|-\sum_{i}\left|R_{i}\right|\right)+r-1=r-1,
\end{aligned}
$$

because $|S|=\sum_{i}\left|R_{i}\right|$.

Claim 5.10. Suppose a set $S \neq V$ contains exactly three special children $R_{1}, R_{2}, R_{3}$ and $|D(S)| \geq 3$. Then $S$ is a special set.

Proof. Note that

$$
|\delta(S)|=\left|\delta\left(R_{1}\right)\right|+\left|\delta\left(R_{2}\right)\right|+\left|\delta\left(R_{3}\right)\right|-2|D(S)|=3+3+3-2|D(S)|=9-2|D(S)| .
$$

Since $S \neq V$, we have $|\delta(S)| \geq 2$ by Claim 5.8. As $|D(S)| \geq 3$, the only possibility is that $|D(S)|=3$ and $|\delta(S)|=3$, which satisfies the first property of a special set. Also, we have $x^{*}(\delta(S))=x^{*}\left(\delta\left(R_{1}\right)\right)+x^{*}\left(\delta\left(R_{2}\right)\right)+x^{*}\left(\delta\left(R_{3}\right)\right)-2 x^{*}(D(S))$. As each term on the right-hand side is an integer, it follows that $x^{*}(\delta(S))$ is an integer. Since $\delta(S) \cap F=\varnothing$, we have $x^{*}(\delta(S))<|\delta(S)|=3$ and thus $x^{*}(\delta(S))$ is either equal to one or two, and so the second property of a special set is satisfied. Finally, note that

$\chi(\delta(S))=\chi\left(\delta\left(R_{1}\right)\right)+\chi\left(\delta\left(R_{2}\right)\right)+\chi\left(\delta\left(R_{3}\right)\right)+2 \chi\left(E^{\prime}\left(R_{1}\right)\right)+2 \chi\left(E^{\prime}\left(R_{2}\right)\right)+2 \chi\left(E^{\prime}\left(R_{3}\right)\right)-2 \chi\left(E^{\prime}(S)\right)$.

Here, the vector $\chi\left(E^{\prime}\left(R_{i}\right)\right)$ will be the zero vector if $R_{i}$ is a special vertex. Since $R_{1}, R_{2}, R_{3}$ satisfy the third property of a special set, $S$ satisfies the third property of a special set. 
Consider the following cases for the induction step.

(1) $S$ contains at least four children. In this case, the induction hypothesis implies that each child has at least one excess token. Therefore, $S$ can collect at least four tokens by taking one excess token from each child. Since $S$ needs two tokens, the other two tokens are extra.

(2) $S$ has exactly three children. If any child has at least two excess tokens, then $S$ can collect four tokens, and we are done. Otherwise, each child has only one excess token, and thus is special by the induction hypothesis. If $S=V$, then $S$ can collect three tokens, and this is enough since $V$ is the root of the laminar family. Else, we have $x^{*}(D(S))=2$ from Claim 5.9. Since there is no edge $e \in D(S)$ with $x_{e}^{*}=1$, we must have $|D(S)|>x^{*}(D(S))=2$. Now, it follows from Claim 5.10 that $S$ is special and it only requires three tokens.

(3) $S$ contains exactly two children $R_{1}, R_{2}$. If both $R_{1}, R_{2}$ have at least two excess tokens, then $S$ can collect four tokens, and we are done. Otherwise, one of the children has exactly one excess token, say $R_{1}$. Hence, $R_{1}$ is special by the induction hypothesis. We now show a contradiction to the independence of tight constraints defining $x^{*}$, and hence this case would not happen.

Since $S$ contains two children, Claim 5.9 implies that $x^{*}(D(S))=1$. Since there is no edge $e \in D(S)$ with $x_{e}^{*}=1$, we have $|D(S)|=\left|\delta\left(R_{1}, R_{2}\right)\right| \geq 2$. Also, $R_{1}$ is special and thus $\left|\delta\left(R_{1}\right)\right|=3$. We claim $\delta\left(R_{1}, R_{2}\right)=\delta\left(R_{1}\right)$. If not, then let $e=\delta\left(R_{1}\right) \backslash \delta\left(R_{1}, R_{2}\right)$. Then

$$
x_{e}^{*}=x^{*}\left(\delta\left(R_{1}\right)\right)-x^{*}\left(\delta\left(R_{1}, R_{2}\right)\right)=x^{*}\left(\delta\left(R_{1}\right)\right)-x^{*}(D(S)) .
$$

But $x^{*}\left(\delta\left(R_{1}\right)\right)$ is an integer as $R_{1}$ is special and $x^{*}(D(S))=1$. Therefore, $x_{e}^{*}$ is an integer which is a contradiction. Thus, $\delta\left(R_{1}, R_{2}\right)=\delta\left(R_{1}\right)$. But then

$$
\chi\left(E^{*}(S)\right)=\chi\left(E^{*}\left(R_{1}\right)\right)+\chi\left(\delta\left(R_{1}\right)\right)+\chi\left(E^{*}\left(R_{2}\right)\right)
$$

if $R_{2} \in \mathcal{L}$ or

$$
\chi\left(E^{*}(S)\right)=\chi\left(E^{*}\left(R_{1}\right)\right)+\chi\left(\delta\left(R_{1}\right)\right)
$$

if $R_{2}$ is a singleton vertex. $R_{1}$ is special implies that $\chi\left(\delta\left(R_{1}\right)\right)$ is a linear combination of the characteristic vectors of its descendants and the characteristic vectors $\left\{\chi(\delta(v)): v \in R_{1} \cap X\right\}$. Hence, in either case, $\chi\left(E^{*}(S)\right)$ is spanned by $\chi\left(E^{*}(R)\right)$ for $R \in \mathcal{L} \backslash\{S\}$ and $\chi(\delta(v))$ for $v \in S \cap X$ which is a contradiction to the linear independence of the constraints in $\mathcal{L}$.

This completes the proof of Lemma 5.7 and Theorem 5.1.

\section{CONCLUDING REMARKS AND OPEN QUESTIONS}

In this article we extend the iterative rounding framework to obtain the best possible guarantee for the MBDST problem. This built on the integrality proofs of the spanning tree polytope. The iterative rounding framework can be used to obtain new proofs of integrality of linear programming formulations for many combinatorial optimization problems including matchings in bipartite and general graphs, matroid bases and matroid intersection, submodular flows, etc. While the proof ideas are similar to other general techniques like total dual integrality, the new iterative proofs are crucial for problems which can be modeled by introducing extra side constraints, for example the degree constraints in the minimum bounded degree spanning tree problem. We refer the reader to Singh [2008] and Lau et al. [2011] for further applications of the technique.

A closely related problem is the well-studied travelling salesperson problem (TSP). The sub-tour elimination relaxation for TSP is very similar to the LP relaxation for the 
MBDST problem. Indeed, our techniques can be used to give the following polyhedral result: Any solution to the sub-tour elimination polytope can be written as a convex combination of 1-trees each of maximum degree three and average degree two, improving on a similar result of Goemans [2006]. Here, a 1-tree is a tree on $V \backslash v$ along with any two edges incident at vertex $v$. A natural open question is whether the techniques used here can be used to obtain better approximation algorithm for TSP.

\section{ACKNOWLEDGMENTS}

The collaboration started when the authors visited Microsoft Research in Redmond; we are very grateful to the Theory Group for their hospitality. M. Singh would like to thank R. Ravi for introducing him to the MBDST problem and innumerable discussions over the past few years. We also thank R. Ravi for help in the preparation of this article.

\section{REFERENCES}

N. Bansal, R. Khandekar, and V. Nagarajan. 2008. Additive guarantees for degree bounded directed network design. In Proceedings of STOC. 769-778.

K. Chaudhuri, S. Rao, S. Riesenfeld, and K. Talwar. 2009a. A push-relabel approximation algorithm for approximating the minimum-degree MST problem and its generalization to matroids. Theoret. Comput. Sci. 410, 44, 4489-4503.

K. Chaudhuri, S. Rao, S. Riesenfeld, and K. Talwar. 2009b. What would Edmonds Do? Augmenting paths and witnesses for degree-bounded MSTs. Algorithmica 55, 1, 157-189.

J. Cheriyan, S. Vempala, and A. Vetta. 2006. Network design via iterative rounding of setpair relaxations. Combinatorica 26, 3, 255-275.

G. Cornuéjols, J. Fonlupt, and D. Naddef. 1985. The traveling salesman problem on a graph and some related integer polyhedra. Math. Program. 33, 1-27. 10.1007/BF01582008.

W. H. Cunningham. 1984. Testing membership in matroid polyhedra. J. Combinat. Theory, Seri. B 36, 2 , 161-188.

J. Edmonds. 1971. Matroids and the greedy algorithm. Math. Program. 1, 127-136. 10.1007/BF01584082.

L. Fleischer, K. Jain, and D. P. Williamson. 2006. Iterative rounding 2-approximation algorithms for minimum-cost vertex connectivity problems. J. Comput. Syst. Sci. 72, 5, 838-867.

M. Fürer and B. Raghavachari. 1994. Approximating the minimum-degree Steiner tree to within one of optimal. J. Algor. 17, 3, 409-423.

M. R. Garey and D. S. Johnson. 1979. Computers and Intractability: A Guide to the Theory of NPCompleteness. W. H. Freeman \& Co., New York.

M. X. Goemans. 2006. Minimum bounded degree spanning trees. In Proceedings of the 47th Annual IEEE Symposium on Foundations of Computer Science. 273-282.

M. Grötschel, L. Lovász, and A. Schrijver. 1981. The ellipsoid method and its consequences in combinatorial optimization. Combinatorica 1, 2, 169-197.

K. Jain. 2001. A Factor 2 approximation algorithm for the generalized steiner network problem. Combinatorica 21, 1, 39-60.

J. Könemann and R. Ravi. 2002. A matter of degree: Improved approximation algorithms for degree-bounded minimum spanning trees. SIAM J. Comput. 31, 6, 1783-1793.

J. Könemann and R. Ravi. 2005. Primal-dual meets local search: Approximating MSTs with nonuniform degree bounds. SIAM J. Comput. 34, 3, 763-773.

L. C. Lau, J. Naor, M. R. Salavatipour, and M. Singh. 2009. Survivable network design with degree or order constraints. SIAM J. Comput. 39, 3, 1062-1087.

L. C. Lau, R. Ravi, and M. Singh. 2011. Iterative Methods in Combinatorial Optimization. Cambridge Tects in Applied Mathematics, Cambridge University Press.

L. C. Lau and M. Singh. 2008. Additive approximation for bounded degree survivable network design. In Proceedings of STOC. 759-768.

R. Ravi, M. V. Marathe, S. S. Ravi, D. J. Rosenkrantz, and H. B. Hunt. 1993. Many birds with one stone: Multiobjective approximation algorithms. In Proceedings of the 25th Annual ACM Symposium on Theory of Computing. 438-447. 
R. Ravi and M. Singh. 2006. Delegate and conquer: An LP-based approximation algorithm for minimum degree MSTs. In Proceedings of the 33rd International Colloquium on Automata, Languages and Programming. 169-180.

A. Schrijver. 2003. Combinatorial Optimization - Polyhedra and Efficiency. Springer.

M. Singh. 2008. Iterative Methods in Combinatorial Optimization. Ph.D. thesis Carnegie Mellon University.

V. V. Vazirani. 2004. Approximation Algorithms. Springer.

S. Win. 1989. On a connection between the existence OFK-trees and the toughness of a graph. Graphs and Combinatorics 5, 1, 201-205.

Received April 2013; revised February 2014; accepted April 2014 